Service social

\title{
Les récents recours à des commissions d'enquête dans le secteur de la santé et des services sociaux au Québec
}

\section{Pierre Bergeron et Vincent Lemieux}

Volume 41, numéro 2, 1992

Bilan des réformes

URI : https://id.erudit.org/iderudit/706567ar

DOI : https://doi.org/10.7202/706567ar

Aller au sommaire du numéro

Éditeur(s)

École de service social de l'Université Laval

ISSN

1708-1734 (numérique)

Découvrir la revue

Citer cet article

Bergeron, P. \& Lemieux, V. (1992). Les récents recours à des commissions d'enquête dans le secteur de la santé et des services sociaux au Québec. Service social, 41(2), 7-17. https://doi.org/10.7202/706567ar
Résumé de l'article

Les auteurs discutent des processus sociopolitiques dans lesquels s'insèrent les commissions Castonguay-Nepveu et Rochon pour cerner la contribution de ces commissions d'enquête québécoises au secteur de la santé et des services sociaux. Ils soulignent ainsi que ces commissions constituent essentiellement des épisodes particuliers de formulation de politiques publiques et qu'elles sont liées aux choix de politiciens. Ils soutiennent également que les rapports de ces commissions et leurs suites traduisent autant l'évolution des interventions publiques québécoises qu'elles ne la déterminent. 


$$
A \quad R I I C C L E S
$$

\section{Les récents recours à des commissions d'enquête dans le secteur de la santé et des services sociaux au Québec}

Pierre BERGERON

Médecin spécialiste Département de santé communautaire Centre hospitalier de l'Université Laval Professeur invité, Département de management Université Laval

Vincent LEMIEUX Professeur, Département de science politique Université Laval

\section{INTRODUCTION}

La pratique des commissions royales d'enquête nous vient de GrandeBretagne et remonte au début de la monarchie normande, soit au $\mathrm{Xl}^{\mathrm{e}}$ siècle ; cette pratique qui a connu une grande vogue au XIX ${ }^{e}$ siècle a produit notament le rapport de Lord Durham sur les problèmes canadiens en 1838. Les gouvernements coloniaux ont transposé cette même pratique, qui s'est poursuivie jusqu'à nos jours (Bonenfant, 1972).

La loi canadienne autorise la création d'une commission d'enquête «sur toute question touchant le bon gouvernement du Canada ou la gestion 
des affaires publiques» (Loi concernant les enquêtes relatives aux affaires publiques et aux ministères, 1985). La loi québécoise sur les commissions d'enquête est semblable, mais ajoute l'administration de la justice et « quelque matière importante se rattachant à la santé publique ou au bien-être de la population » (Loi sur les commissions d'enquête, 1990).

Tant le gouvernement canadien que le gouvernement québécois ont largement fait appel à ces commissions. Le gouvernement canadien a institué plus de 340 commissions royales d'enquête de 1870 à 1966 (Courtney, 1969 ; Henderson, 1967). De même, le gouvernement québécois a institué plus de 50 commissions ou comités d'enquête de 1867 à 1971 (Bonenfant, 1972).

La Commission de réforme du droit du Canada distingue deux grands types de commissions d'enquête (Commission de réforme du droit du Canada, 1979). Un premier vise à résoudre des controverses administratives ou politiques par la production d'un avis impartial, indépendant des partis politiques et du gouvernement. De telles commissions ont été généralement confiées à des juges et ont été mises sur pied à la suite de cas de corruption, de désastres ou d'accidents majeurs. Régulièrement utilisé au siècle dernier, ce premier type a progressivement perdu de l'importance par rapport au second, qui prédomine actuellement (Aucoin, 1987).

Le second type de commission d'enquête vise la réalisation de vastes enquêtes sur des enjeux essentiels liés aux politiques publiques. De telles commissions se composent de personnes qui viennent surtout du monde politique, universitaire ou des affaires, et qui reçoivent l'aide d'équipes d'experts. Depuis les années 60, leurs mandats ont couvert tous les grands secteurs d'activité de notre société (Aucoin, 1987).

Dans le secteur de la santé et des services sociaux, le gouvernement québécois a créé de telles commissions à deux reprises au cours des vingtcinq dernières années. Les travaux de la commission sur la santé et le bienêtre social (Castonguay-Nepveu) se sont déroulés de 1966 à 1972, les travaux de la seconde, sur les services de santé et les services sociaux (Rochon), de 1985 à 1988. Toutes deux s'inscrivent dans des épisodes majeurs de révision des politiques publiques dans ce secteur. Comme les travaux de ces commissions ont précédé les réformes législatives de 1971 et de 1991, plusieurs tendent à leur attribuer une contribution déterminante dans l'évolution des interventions publiques.

Ce texte discute des processus sociopolitiques dans lesquels s'insèrent les commissions Castonguay-Nepveu et Rochon pour mieux cerner leur contribution au secteur de la santé et des services sociaux. Il souligne ainsi que ces commissions constituent essentiellement des épisodes particuliers de formulation de politiques publiques et qu'elles sont liées aux choix de politiciens. Il soutient également que les rapports de ces commissions et 
leurs suites traduisent autant l'évolution des interventions publiques québécoises qu'elles ne la déterminent.

\section{LA FORMULATION DE POLITIQUES PUBLIQUES ET LES ÉPISODES CASTONGUAY-NEPVEU ET ROCHON}

On distingue souvent, dans l'analyse des politiques, le processus d'émergence, de formulation, d'adoption et de mise en œuvre. Cette distinction n'atténue en rien les multiples chevauchements et recoupements de ces processus. Par contre, elle identifie des enjeux différents et permet d'associer les commissions d'enquête avec l'enjeu particulier de la formulation de politiques publiques. En relation avec cet enjeu, les acteurs font principalement appel à des idées ou à des schèmes de représentation pour formuler des politiques.

Les commissions d'enquête sont des épisodes, car elles désignent des pratiques clairement délimitées dans l'espace et dans le temps. Ces épisodes sont particuliers parce qu'ils renvoient à la loi québécoise sur les commissions d'enquête ainsi qu'à l'arrêté en conseil établissant un mandat, une composition, un échéancier et un budget. De même, ces épisodes sont distincts d'épisodes subséquents ou concurrents portant sur l'adoption ou la mise en œuvre de politiques publiques. Enfin, les commissions d'enquête désignent des pratiques distinctes des autres interventions publiques qui se poursuivent parallèlement à leurs travaux.

Sur le plan de la formulation des politiques publiques, les commissions d'enquête ne sont qu'un des modes possibles ou qu'une des modalités auxquelles recourent les politiciens. Ainsi Trebilcock et ses collaborateurs associent ces commissions aux autres modalités d'enquête publique que sont les groupes de travail, les commissions parlementaires, les enquêtes par des organismes-conseils ou des organismes de contrôle permanents ainsi que les études de groupes ministériels ou interministériels (Trebilcock, 1982).

Ces modes d'enquête publique autres que les commissions d'enquête donnent évidemment lieu à des épisodes de formulation différents. Ils peuvent mettre à contribution des administrateurs publics, des groupes restreints de conseillers ou encore des élus. Ils peuvent avoir ou non un caractère public et comporter des chevauchements entre la formulation et I'adoption de leurs propositions. La coexistence et la concurrence de ces autres modes de formulation montrent bien que les commissions d'enquête sont des épisodes particuliers qui s'insèrent dans la formulation et la révision de politiques publiques.

Ainsi la révision des politiques publiques amenée par la création de la Commission Rochon en 1985 se poursuit sous diverses formes avec la publication du document Orientations de la ministre Lavoie-Roux, puis avec 
un avant-projet de loi, une commission parlementaire, un livre blanc présentant la réforme Côté et une autre commission parlementaire. Par la suite, la contestation du projet de loi 120 par les médecins entraîne le ministre Côté dans des négociations avec ces derniers avant que I'Assemblée nationale n'adopte cette loi en août 1991.

Mais là encore, la formulation des politiques à l'égard du financement du système de santé et de services sociaux demeure en suspens. Suivent alors un livre blanc et une autre commission parlementaire, qui précèdent les compressions budgétaires et les désassurances annoncées dans le budget provincial 1992-1993. Il apparaît ainsi très clairement que la Commission Rochon ne constitue qu'un épisode particulier de la formulation de ces politiques publiques.

Il en est de même pour la révision précédente. Tout d'abord, la création de la Commission Castonguay-Nepveu fait suite au Comité d'étude sur l'assurance-maladie. De plus, cette commission se retrouve sur une voie secondaire dès 1971, c'est-à-dire avant la fin de ses travaux. Cette mise à I'ombre tient à l'accession de son président au poste de ministre responsable des affaires sociales. Ce dernier conserve alors l'initiative et formule, avec les hauts fonctionnaires de son nouveau ministère, un ensemble de propositions législatives qui participent à la profonde réforme de ce secteur (Bergeron, 1990).

\section{LE CHOIX POLITIQUE DE RECOURIR AUX COMMISSIONS CASTONGUAY-NEPVEU ET ROCHON}

Par ailleurs, ces épisodes que sont les commissions Castonguay-Nepveu et Rochon sont eux-mêmes liés à un choix politique antérieur, celui de recourir à une commission d'enquête pour réviser les politiques publiques. Les différents modes de formulation ou d'enquête publique constituent en effet des instruments d'intervention dont disposent les politiciens dans notre système démocratique canadien (Wilson, 1971).

Les politiciens font appel à ces instruments un peu comme ils le font pour d'autres instruments comme la nationalisation, l'imposition, les dépenses publiques, la gestion de la dette ou la réglementation. Ils peuvent ainsi recourir aux enquêtes publiques à diverses fins particulières.

In sum, all public inquiries offer to politicians the opportunity to demonstrate concern about a policy issue and to indicate that action is being taken upon it, while deferring the need to expend substantial resources in response to a perceived policy concern; to obtain advice, while maintaining some distance from the source of that advice, so as to be able to reject it where necessary ; to generate, analyse, and exchange information, while informing and persuading 
the public of the merits of certain points of view; and to assess the public's tastes for proposed policy initiatives (Trebilcock, 1982 : 44).

Le choix politique d'un mode ou l'autre prend en considération leurs traits institutionnels différents, comme le caractère privé ou public de leurs travaux, les relations avec le ministre ou le gouvernement et la nature du mandat. Mais il tient également à la composition différente des groupes, comités ou commissions. Selon cette composition, différentes catégories $\mathrm{d}^{\prime}$ acteurs seront plus ou moins habilitées et seront en plus ou moins bonne position pour faire valoir leurs préférences.

Dans le cas des commissions d'enquête, les acteurs les plus habilités sont ceux du monde politique, universitaire ou des affaires ainsi que les experts. On peut alors se demander pourquoi des élus québécois ont choisi de recourir à une commission à deux reprises à vingt ans d'intervalle pour formuler des politiques publiques. Ces recours à une commission paraissent largement liés à de fortes exigences quant à la légitimité des interventions publiques dans le secteur de la santé et des services sociaux.

Ces interventions sont en effet délicates en raison de la complexité et de la sensibilité politique d'un secteur caractérisé par un très large éventail de services et par les pratiques de multiples groupes d'acteurs; de plus, ce secteur représente des enjeux concrets pour l'ensemble de la population et draine une part importante des ressources collectives.

Une commission d'enquête peut alors renforcer la légitimité politique d'éventuelles interventions en associant diverses personnalités au processus de formulation de politiques publiques. En même temps, ce mode permet aux politiciens de gagner du temps ; il leur permet $d^{\prime}$ agir sans vraiment intervenir et de conserver leur marge de manœuvre.

Or, la création des commissions Castonguay-Nepveu et Rochon appartient effectivement à des gouvernements relativement faibles, le dernier gouvernement de l'Union nationale en 1966 et le gouvernement du Parti québécois, déchiré et en fin de mandat, en 1985. À l'opposé, les deux gouvernements libéraux qui héritent de ces commissions d'enquête disposent de mandats électoraux forts ; ils peuvent se permettre de resituer les travaux de ces commissions d'enquête au second plan, mais évitent d'y mettre fin. Ainsi, dans le cas de Castonguay-Nepveu, le gouvernement libéral de 1971 démarre immédiatement une réforme, alors que dans le cas de Rochon le gouvernement libéral de 1985 revoit sa composition et son mandat.

Le recours aux commissions d'enquête vise également à assurer une plus grande légitimité scientifique aux interventions publiques. En effet, le mode d'une telle commission fait largement appel aux experts et paraît favoriser I'utilisation des connaissances scientifiques dans la prise de décision gouvernementale (Aucoin, 1987 ; Courtney, 1969). Ainsi certains analystes estiment que les commissions d'enquête sont des instruments 
particulièrement utiles pour les gouvernements dans des périodes de crise ou lorsqu'ils font face à des problèmes apparemment inextricables.

Aucoin les considère même comme le mécanisme le plus efficace pour assurer l'indépendance et l'objectivité dans la révision des politiques publiques (Aucoin, 1987). Cette perspective de gestion gouvernementale s'accorde avec la régulation étatique centrale qui est visée à la fin des années 60 ; de même, au milieu des années 80 elle s'accorde avec le souci de renforcer une telle régulation (Bergeron, 1990).

Le secteur de l'éducation paraît fournir la contrepartie de cet argument. Dans ce secteur, la forte prédominance de la régulation administrative centrale héritée de la réforme Parent dans les années 60 contribuerait à expliquer l'absence de commissions d'enquête subséquentes. Les tensions entre les groupes, le financement, le rôle de l'État n'apparaissent pas au premier plan des débats ; ces derniers s'inscrivent davantage à l'intérieur de la rationalité administrative et portent plutôt sur les programmes, leur contenu, leur complémentarité et leur enchaînement.

Par exemple, le traitement de la clientèle ne se présente pas de la même façon dans le secteur de la santé et des services sociaux que dans le secteur de l'éducation. Dans ce dernier secteur, les clients, du primaire au secondaire, du secondaire au collégial et du collégial à l'universitaire, suivent des séquences. Une réforme du secteur, qui touche de façon marquée une des étapes, a des conséquences importantes sur d'autres étapes. Si l'on décidait de supprimer les cégeps et $d^{\prime}$ allonger $d^{\prime} u n$ an le secondaire et l'universitaire, les bouleversements dans le secteur de l'éducation seraient considérables.

II n'en est pas de même dans le secteur de la santé et des services sociaux, où le traitement des clients n'est pas soumis à la même progression. Ainsi la suppression des Centres de services sociaux n'entraîne pas des bouleversements aussi considérables que ceux que pourrait provoquer la suppression des cégeps.

Au-delà du recours au mode particulier des commissions d'enquête, la fréquence des révisions majeures des politiques publiques dans le secteur de la santé et des services sociaux peut quand même paraître surprenante. À cet égard, il semble que cette fréquence soit elle-même liée au rythme de transformation propre à un secteur particulièrement important, ne serait-ce que parce qu'il draine une forte part du PIB. L'évolution du secteur québécois de la santé et des services sociaux montre la prise en charge étatique de larges secteurs sociaux dans les années 60 et la mise en cause de cette même prise en charge dans les années 80 .

L'évolution des interventions au Québec n'est pas coupée des transformations des systèmes de santé et de services sociaux dans les autres sociétés, dont ceux des provinces canadiennes et de plusieurs sociétés occidentales industrialisées (OCDE, 1988). Cette évolution parallèle 
s'applique encore à la situation actuelle; le gouvernement québécois modifie son système au même moment où plusieurs provinces canadiennes (Angus, 1990) et plusieurs pays européens le font.

\section{LA PRODUCTION ET LES SUITES DES RAPPORTS CASTONGUAY-NEPVEU ET ROCHON}

$L^{\prime}$ insertion de la formulation des politiques publiques dans un contexte politique et social plus large s'applique à la production des rapports Castonguay-Nepveu et Rochon ainsi qu'à leurs suites respectives. Le contenu d'un tel rapport est produit par un ensemble d'acteurs engagés dans les travaux des commissions. Pour le cas de la commission Rochon, nous avons montré que différents groupes ou alliances d'acteurs privilégient différentes politiques publiques (Lemieux, 1989 ; Bergeron, 1992). Selon notre analyse, ces alliances s'efforcent d'accentuer, dans les politiques proposées, l'un ou l'autre des grands traits institutionnels que sont la surveillance de l'État, l'appartenance communautaire, les jeux partisans, l'individualisme et le corporatisme. L'étude du cas de la Commission Rochon met ainsi en lumière que les préférences de l'alliance bureaucratique ou socio-étatique ont largement prévalu dans la production de son rapport (Bergeron, 1992).

Dans son analyse de l'interaction entre des alliances d'acteurs, cette étude révèle également comment le déroulement de cette commission est indissociable de son contexte. Ainsi, la promotion de différentes politiques par les alliances d'acteurs se réalise nécessairement en relation avec les pratiques en cours dans le secteur de la santé et des services sociaux. Le rapport de la Commission Rochon ne peut pas faire abstraction du caractère public et relativement hiérarchique du système en place, ni des champs d'auto-nomie dont disposent des groupes comme les médecins. Les auteurs du rapport en tiennent compte même lorsqu'ils les dénoncent ou proposent de les changer.

De plus, les auteurs du rapport sont jusqu'à un certain point contraints par les schèmes de représentation qui prévalent à l'égard du secteur concerné. L'échéancier relativement restreint d'une commission, la recherche du consensus parmi les commissaires, la prédominance d'approches théoriques particulières dans les milieux de recherche tendent à limiter la production de nouvelles connaissances par les commissions. Ces contraintes favorisent une mise en forme nouvelle de voies de solutions existantes plutôt que le renouvellement et l'innovation (Bergeron, 1992 ; Aucoin, 1987 ; Simeon, 1987).

De même, les suites aux rapports des commissions tiennent beaucoup à la concordance de leur contenu avec le contexte sociopolitique dans lequel ces rapports s'insèrent. La contribution des deux commissions 
d'enquête à l'évolution des politiques publiques est certes liée aux personnes qui ont produit et fait valoir le contenu de leurs rapports; mais elle reflète encore plus le contexte social et politique dans lequel la diffusion de ces rapports s'est insérée.

Ainsi, les répercussions profondes du rapport de la Commission Castonguay-Nepveu tiennent largement au contexte particulier d'une société québécoise en transformation. La prise en charge étatique de ce secteur proposée par la Commission, qui, dans le projet de loi 65, devient un nouveau compromis associant une régulation administrative de l'État avec le maintien de l'autonomie, en particulier, des groupes de médecins et d'hôpitaux, s'accorde avec la société québécoise du début des années 70.

Par contre, la reconduction des solutions Castonguay-Nepveu que propose le rapport de la Commission Rochon colle beaucoup moins à la société québécoise de la fin des années 80 (Bergeron, 1990). La révision des politiques publiques déclenchée en 1985 vise à trouver un nouveau compromis entre le maintien d'un système public et la montée de l'entreprise privée qui favorise le désengagement de l'État. En proposant le renforcement de la régulation administrative de l'État, la Commission Rochon n'offre pas ce compromis, mais reproduit la tension profonde à l'origine même du processus de révision de politiques publiques dans ce secteur (Bergeron, 1992).

Enfin, la portée des rapports de ces commissions d'enquête tient également au fait que ces épisodes de formulation de politiques publiques sont distincts des épisodes subséquents, avec des pratiques et des acteurs différents. Les rapports des commissions d'enquête font, entre autres, largement appel aux travaux d'experts, dont l'influence est souvent restreinte dans les phases ultérieures de la formulation et au moment de l'adoption des politiques publiques. Les propositions de politiques publiques que contiennent les rapports des commissions d'enquête ne conduisent pas directement aux réformes législatives qui sont ultérieurement adoptées.

L'adoption des politiques publiques est un processus différent qui renvoie à des épisodes nettement distincts de leur formulation. Ce ne sont pas les experts et les membres de la Commission qui ont eu l'avantage dans les débats qui ont finalement mené à l'adoption du projet de loi 120. Ce sont plutôt les ministres et leurs entourages partisans ou administratifs, et des groupes d'intéressés puissants, dont en particulier les groupes de médecins. Il en est d'ailleurs de même de la plupart des politiques publiques, dans le secteur de la santé et des services sociaux ou dans d'autres secteurs.

La portée limitée des contributions des commissions d'enquête tient aussi au caractère restreint des politiques qu'elles formulent. Dans leur sillage, les réformes législatives de 1971 et 1991, tout importantes qu'elles aient été, ne couvrent qu'un volet des interventions publiques. En effet, la Loi sur les services de santé et les services sociaux de 1971 porte principalement sur les 
pouvoirs du ministre ainsi que sur la formation et les pouvoirs des conseils régionaux et des établissements. De même, la révision complète de cette loi, en 1991, concerne encore essentiellement la répartition des pouvoirs et responsabilités pour les différents organismes et établissements de ce secteur. Or, beaucoup d'autres pratiques gouvernementales constituent des volets, explicites ou implicites, des interventions de l'État et elles ont des répercussions profondes sur ce secteur.

Les interventions publiques dans un domaine donné englobent aussi bien la priorité donnée à certains objectifs sociaux et la formalisation de cadres organisationnels que des allocations de ressources ou des stratégies de financement. II faut ainsi prendre en considération les sources de financement et les compressions budgétaires, le contenu des conventions collectives avec les multiples syndicats professionnels ou autres, les modifications de la couverture des programmes d'assurance-maladie, ou encore les immobilisations majeures pour I’hébergement et les soins prolongés.

Ces interventions prennent également la forme de stratégies administratives et de choix privilégiant certaines clientèles. Plusieurs documents ministériels ou gouvernementaux témoignent de ces choix et de ces priorités, tels la Politique de maintien à domicile (1979), Un nouvel âge à partager (politique sur les personnes âgées), I'arrêté en conseil 3179-79 sur la décentralisation administrative vers les CRSSS, la Politique sur le parachèvement du réseau des CLSC, la Politique d'aide aux femmes violentées (1985) ou encore le Plan de désengorgement des urgences des centres hospitaliers (1986). De même, plusieurs interventions législatives s'ajoutent à la Loi sur les services de santé et les services sociaux, comme la Loi de I'adoption (1969), la Loi assurant l'exercice des droits des personnes handicapées (1978), la Loi sur la protection de la jeunesse (1979) et la Loi canadienne sur les jeunes contrevenants (1984).

\section{CONCLUSION}

En conclusion, la prise en considération du contexte sociopolitique fournit un éclairage indispensable pour analyser la contribution des commissions d'enquête à l'évolution des politiques publiques dans le secteur de la santé et des services sociaux au Québec. Tout en reconnaissant l'importance des acteurs engagés et des pratiques institutionnalisées dans le secteur concerné, nous avons insisté sur l'insertion des commissions d'enquête dans des processus sociaux et politiques plus larges, parce que cette insertion nous semble trop souvent négligée.

Ces processus sociaux rejoignent tant la recherche et l'enseignement que I'administration publique et les stratégies de divers groupes sociaux. 
Les commissions d'enquête ont ainsi des répercussions multiples sur de nombreux systèmes sociaux qui débordent leur contexte particulier et participent de la reproduction et de la transformation de la société québécoise.

En même temps, il ne faut pas sous-estimer la valeur symbolique des commissions d'enquête comme instrument de légitimation de politiques publiques qui n'ont pas nécessairement de rapport étroit avec ce qui avait été recommandé par ces commissaires. Contrairement à d'autres actions liées à la réalisation des politiques publiques, les commissions d'enquête font l'objet de beaucoup de publicité, elles consultent beaucoup de groupes et de personnes et elles reçoivent les avis de nombreux experts. Les rapports des commissions d'enquête peuvent alors servir autant à légitimer I'adoption de certaines politique publiques qu'à alimenter cette adoption par leurs recommandations.

\section{Références bibliographiques}

ANGUS, Douglas E. (1990). A Great Canadian Prescription : Take Two Commissioned Studies and Call Me in the Morning (IVe Conférence canadienne sur l'économie de la santé, Université de Toronto, 27-29 août), Canadian Hospital Association, $36 \mathrm{p}$.

AuCOIN, Peter (1987). "Royal Commissions and Task Forces as Mechanisms of Program Review », The Canadian Journal of Program Evaluation, vol. 2, $\mathrm{n}^{\circ} 2: 1-10$.

BERGERON, Pierre (1990). "La commission Rochon reproduit les solutions de Castonguay-Nepveu », Recherches sociographiques, vol. XXXI, n 3 : 359380.

BERGERON, Pierre (1992). La commission Rochon sous le prisme des alliances, thèse de doctorat, Département de science politique, Faculté des sciences sociales, Université Laval, 238 p. + x.

BONENFANT, Jean-Charles (1972). "Les commissions d'enquête du Québec », Annuaire du Québec 1972. Québec : Bureau de la statistique du Québec, ministère de I'Industrie et du Commerce, p. 36-37.

CANADA (1985). "Loi concernant les enquêtes relatives aux affaires publiques et aux ministères », Lois révisées du Canada (1985), vol. 5, c. I-II, art. 2. Ottawa : Imprimeur de la Reine.

COMMISSION DE RÉFORME DU DROIT DU CANADA (1979). Rapport sur les commissions consultatives et les commissions d'enquête, $13^{\mathrm{e}}$ rapport, ministère des Approvisionnements et Services Canada, $51 \mathrm{p}$.

COURTNEY, John C. (1969). "In Defence of Royal Commissions », Administration publique du Canada, vol. 12, nº 2 : 198-212.

Henderson, G.F. (1967). Federal Royal Commissions in Canada, 1867-1966 : A Checklist. Toronto : University of Toronto Press.

LEMIEUX, Vincent (1989). "La Commission Rochon et la réalisation des politiques publiques », Administration publique du Canada, vol. 32, nº 2 : 261-273. 
ORGANISATION DE COOPÉRATION ET DE DÉVELOPPEMENT ÉCONOMIQUES [OCDE] (1988). L'avenir de la protection sociale, Études de politique sociale, $\mathrm{n}^{\circ} 6$. Paris : Éditions de l'OCDE, $62 \mathrm{p}$.

QUÉBEC (1990). "Loi sur les commissions d'enquête », Lois refondues du Québec, c. C-37, art. 1. Québec : Éditeur officiel du Québec.

SIMEON, Richard (1987). "Inside the Macdonald Commission », Studies in Political Economy, vol. 22 : 167-179.

Trebilcock, M.J., D.G. HARTLE, R.S. PriChard, D.N. DeWEes (1982). The Choice of Governing Instrument, A study prepared for the Economic Council of Canada, Minister of Supply and Services Canada, chap. 3-4 : 21-50.

WILSON, V. Seymour (1971). "The Role of Royal Commissions and Task Forces», dans Bruce G. Doern et Peter Aucoin (ed.), The Structures of Policy-Making in Canada.Toronto : Macmillan of Canada, chap. 4 : 113-129. 